\title{
Increased Default Mode Network Connectivity in Individuals at High Familial Risk for Depression
}

\author{
Jonathan Posner",1,2, Jiook Cha ${ }^{1,2}$, Zhishun Wang ${ }^{1,2}$, Ardesheer Talati ${ }^{1,2}$, Virginia Warner ${ }^{1,2}$, \\ Andrew Gerber ${ }^{1,2}$, Bradley S Peterson ${ }^{3}$ and Myrna Weissman ${ }^{1,2}$ \\ 'Department of Psychiatry, College of Physicians and Surgeons, Columbia University, New York, NY, USA; ${ }^{2}$ New York State Psychiatric Institute, \\ New York, NY, USA; ${ }^{3}$ Institute for the Developing Mind, Children's Hospital Los Angeles and the Keck School of Medicine, University of Southern \\ California, Los Angeles, CA, USA
}

\begin{abstract}
Research into the pathophysiology of major depressive disorder (MDD) has focused largely on individuals already affected by MDD. Studies have thus been limited in their ability to disentangle effects that arise as a result of MDD from precursors of the disorder. By studying individuals at high familial risk for MDD, we aimed to identify potential biomarkers indexing risk for developing MDD, a critical step toward advancing prevention and early intervention. Using resting-state functional connectivity MRI (rs-fcMRI) and diffusion MRI (tractography), we examined connectivity within the default mode network (DMN) and between the DMN and the central executive network (CEN) in I I I individuals, aged I I-60 years, at high and low familial risk for depression. Study participants were part of a threegeneration longitudinal, cohort study of familial depression. Based on rs-fcMRI, individuals at high vs low familial risk for depression showed increased DMN connectivity, as well as decreased DMN-CEN-negative connectivity. These findings remained significant after excluding individuals with a current or lifetime history of depression. Diffusion MRI measures based on tractography supported the findings of decreased DMN-CEN-negative connectivity. Path analyses indicated that decreased DMN-CEN-negative connectivity mediated a relationship between familial risk and a neuropsychological measure of impulsivity. Our findings suggest that DMN and DMN-CEN connectivity differ in those at high vs low risk for depression and thus suggest potential biomarkers for identifying individuals at risk for developing MDD.

Neuropsychopharmacology (2016) 4I, 1759-1767; doi:I0.1038/npp.2015.342; published online 16 December 2015
\end{abstract}

\section{INTRODUCTION}

Current approaches are effective in treating major depressive disorder (MDD), yet more than half of patients either fail to remit or drop out of treatment prematurely (Rush et al, 2006). Even in those who respond, significant impairments including underemployment, disability, and disproportionate levels of medical illness persist (Kessler et al, 1997; Paradiso et al, 1997; Keller, 2003). An alternative strategy is to identify those at high risk for developing MDD with the goal of advancing prevention and early intervention. Critical to advancing this preventive approach, however, is the identification of biomarkers predictive of the development of MDD.

Research into the pathophysiology of MDD has focused largely on individuals already affected by MDD. Studies have thus been limited in their ability to disentangle effects that arise as a result of the disorder from predictors of the disorder. By focusing our research on individuals at risk for

\footnotetext{
*Correspondence: Dr J Posner, College of Physicians and Surgeons and New York State Psychiatric Institute, Columbia University, Unit 74, I 05 I Riverside Drive, New York, NY I0032, USA, Tel: +646 774 5735, Fax: +314 27| 2446, E-mail: posneri@nyspi.columbia.edu

Received 26 February 2015; revised 5 October 2015; accepted 21 October 2015; accepted article preview online 23 November 2015
}

developing MDD, we have tried to circumvent this limitation (Peterson et al, 2009; Dubin et al, 2012). Toward this end, we used resting-state functional connectivity MRI (rs-fcMRI) to examine the connectivity of the brain's default mode network (DMN) in individuals at high and low familial risk for depression. The DMN is a collection of brain regions that reliably deactivate during goal-directed behaviors (Raichle and Snyder, 2007). We focused on DMN connectivity because prior studies suggest increased DMN connectivity is associated with MDD (Greicius et al, 2007; Sheline et al, 2009). Prior studies, however, have seldom examined whether increased DMN connectivity is an antecedent or consequence of MDD, and thus it remains unknown whether increased DMN connectivity represents a potential biomarker predictive of depression.

We also examined connectivity between the DMN and the central executive network (CEN), encompassing the dorsolateral prefrontal and parietal cortices. The DMN and the CEN habitually work in opposing directions in relation to attentional demands-as attentional demands increase, CEN activation increases while DMN activation decreases; conversely, during periods of rest or internally focused cognitions, CEN activation decreases while DMN activation increases (Raichle et al, 2001; Fox et al, 2005; Grady et al, 2010). This inverse relationship between the DMN and CEN 
is reflected in negative connectivity (or anticorrelations) between these two networks in healthy individuals (Fox et al, 2005; Biswal et al, 2010; Whitfield-Gabrieli and Ford, 2012). DMN-CEN anticorrelations may be altered in MDD (Sheline et al, 2010; Hamilton et al, 2011) and may reflect impairments in attentional control or impulsivity (Posner et al, 2014); however, similar to DMN connectivity, DMN-CEN anticorrelations have scarcely been examined in individuals at risk for, but not yet affected by, depression.

We used rs-fcMRI to examine DMN and DMN-CEN functional connectivity in individuals from a longitudinal cohort study of families at high and low risk for depression. Based on prior literature (Sheline et al, 2009; Posner et al, 2013), we hypothesized that compared with individuals at low familial risk, those at high risk for depression would demonstrate increased connectivity within the DMN, as well as decreased negative connectivity (ie, reduced anticorrelations) between the DMN and CEN. Using diffusion MRI, we then explored whether measures of DMN and DMN-CEN functional connectivity could be corroborated with diffusion tractography. Last, we used path analyses to explore associations between familiar risk for depression, connectivity measures, and affective symptoms and impulsivity.

\section{MATERIALS AND METHODS}

The Institutional Review Board of the New York State Psychiatric Institute (NYSPI) approved the study procedures. Adult participants provided informed consent; minors provided informed assent, and a parent/guardian provided consent.

\section{Participants}

The familial depression study began in 1982; complete details on study design, sample selection, and assessments are reported elsewhere (Weissman et al, 2005). Briefly, risk status for depression was defined based on the first generation (G1), such that offspring (generations 2 and 3, G2 and G3) were defined as high risk if G1 had a history of MDD, and were otherwise defined as low risk. The high- and low-risk families have been followed prospectively for up to 30 years and up to six time points ('Waves') affording exceptional psychiatric assessments of all study participants (Table 1). The current study is based on data collection at Wave 6. Diagnostic interviews were conducted using the Schedule for Affective Disorders and Schizophrenia-Lifetime Version (the adult version for participants over age 18 years, and the child version for participants 6-17 years of age) at up to six time points over up to 30 years. (See Supplementary Materials for further details on assessment procedures.)

We obtained MRI scans from 111 descendants of G1 families, aged 11-60 years. MRI scans from 7 individuals were excluded because of excessive head motion and/or imaging artifacts, leaving 104 individuals available for group comparisons. Of these, 57 participants comprised the highrisk group and 47 the low-risk group. Participants were group matched on sex and age (Table 1). All participants were Caucasian and G1 participants were all drawn from the same community. Exclusion criteria consisted of psychotic symptoms, pregnancy, and MRI contraindications.
Table I Demographic and Clinical Characteristics of Study Participants

\begin{tabular}{|c|c|c|c|c|}
\hline & $\begin{array}{l}\text { High risk } \\
(n=57)\end{array}$ & $\begin{array}{l}\text { Low risk } \\
(n=47)\end{array}$ & $\begin{array}{c}\text { Test } \\
\text { statistic }\end{array}$ & $P$-value \\
\hline Age, mean, years & $34.7 \pm 14.5$ & $29.7 \pm 13.2$ & $t=1.8$ & 0.07 \\
\hline \multicolumn{5}{|l|}{ Age, by generation, years } \\
\hline Second generation (G2) & $46.0 \pm 8.0$ & $48.3 \pm 6.0$ & $t=0.9$ & 0.35 \\
\hline Third generation (G3) & $20.6 \pm 5.1$ & $21.8 \pm 4.5$ & $t=1.0$ & 0.34 \\
\hline \multicolumn{5}{|l|}{ Generation } \\
\hline Second generation (G2) & 31 & 15 & $X^{2}=5.7$ & $0.02^{\mathrm{a}}$ \\
\hline Third generation (G3) & 26 & 33 & & \\
\hline \multicolumn{5}{|l|}{ Gender } \\
\hline Male & 25 & 24 & $X^{2}=0.4$ & 0.53 \\
\hline Female & 32 & 24 & & \\
\hline \multicolumn{5}{|l|}{ Depressive symptoms } \\
\hline Adult & $3.7 \pm 5.3$ & $1.7 \pm 4.5$ & $t=2.0$ & 0.06 \\
\hline Child & $17.9 \pm 0.9$ & $18.8 \pm 3.5$ & $t=0.7$ & 0.49 \\
\hline \multicolumn{5}{|l|}{ Anxiety symptoms } \\
\hline Adult & $3.3 \pm 4.6$ & $1.0 \pm 2.7$ & $t=2.6$ & $0.01^{\mathrm{a}}$ \\
\hline Child & $3.6 \pm 2.4$ & $4.2 \pm 4.3$ & $t=0.3$ & 0.76 \\
\hline $\begin{array}{l}\text { Current or prior } \\
\text { psychotropic medications }\end{array}$ & 12 & 4 & $X^{2}=3.1$ & 0.10 \\
\hline $\begin{array}{l}\text { Current/lifetime depressive } \\
\text { disorder }\end{array}$ & 32 & 12 & $X^{2}=10.4$ & $0.001^{a}$ \\
\hline $\begin{array}{l}\text { Current/lifetime anxiety } \\
\text { disorder }\end{array}$ & 35 & 20 & $X^{2}=4.1$ & $0.04^{\mathrm{a}}$ \\
\hline $\begin{array}{l}\text { Current/substance use } \\
\text { Disorder }\end{array}$ & 18 & 11 & $X^{2}=1.0$ & 0.32 \\
\hline CPT, omission scores & $3.3 \pm 6.9$ & $4.4 \pm 6.3$ & $t=0.8$ & 0.42 \\
\hline CPT, commission scores & $11.8 \pm 8.3$ & $13.7 \pm 8.1$ & $t=1.1$ & 0.37 \\
\hline
\end{tabular}

Note: Depressive symptoms were determined by Hamilton Depression Rating Scale and the Children's Depression Inventory for adults and children, respectively. Anxiety symptoms were determined by the Hamilton Anxiety Rating Scale and the Revised Children's Manifest Anxiety Scale for adults and children, respectively. Values are mean \pm SD unless specified.

${ }^{a}$ Statistical significance.

\section{MRI Pulse Sequences}

Images were acquired on a GE Signa $3.0 \mathrm{~T}$ whole-body scanner using an 8-channel head coil. During resting state acquisition, participants were instructed to remain still with their eyes closed and to let their minds wander freely. Two 9-min resting state scans were obtained for each participant. Diffusion MR images were acquired in two runs with diffusion weighting along 15 non-collinear directions. (Further details on MRI pulse sequences are provided in the Supplementary Materials.)

\section{Resting State fMRI Image Processing}

As described elsewhere (Posner et al, 2013; Posner et al, 2014), standard image preprocessing methods were used, 
employing SPM8 software (http://www.fil.ion.ucl.ac.uk/spm/). Briefly, functional images were slice time and motion corrected, coregistered with a high-resolution anatomical scan, normalized to Montreal Neurological Institute space, resampled at $3 \mathrm{~mm}^{3}$, and smoothed with a Gaussian kernel of $8 \mathrm{~mm}^{3}$ FWHM (Friston et al, 1995). Connectivity processing consisted of independent component analysis (ICA) and a hierarchical partner matching algorithm (Wang et al, 2011) to isolate a network of regions corresponding to the DMN. Partner matching is a clustering algorithm that identifies ICAderived independent components that share spatial properties across subjects (Wang and Peterson, 2008).

To examine the confounding influence of head motion upon connectivity measures, we calculated root mean square and peak/average (across volumes) framewise displacement (FD), which is based on each individual's head alignment parameters from SPM's realignment procedure. We differentiated the six head realignment parameters across frames and then calculated instantaneous head motion as a scalar in each frame using the following formula: $\mathrm{FDi}=\left|\Delta d_{\mathrm{ix}}\right|+\left|\Delta d_{\mathrm{iy}}\right|+\mid$ $\Delta d_{\mathrm{iz}}|+| \Delta \alpha_{\mathrm{i}}|+| \Delta \beta_{\mathrm{i}}|+| \Delta \gamma_{\mathrm{i}} \mid$, where $\Delta d_{\mathrm{ix}}=d_{(\mathrm{i}-1) \mathrm{x}}-d_{\mathrm{ix}}$, and similarly for the other rigid body parameters $\left[d_{\mathrm{ix}} d_{\mathrm{iy}} d_{\mathrm{iz}} \alpha_{\mathrm{i}} \beta_{\mathrm{i}} \gamma_{\mathrm{i}}\right]$. We converted rotational displacements from degrees to millimeters by calculating displacement on the surface of a sphere of radius $50 \mathrm{~mm}$ (Power et al, 2012). Group differences in the motion parameters were tested using the non-parametric Kolmogorov-Smirnov test given nonnormality and existence of potential outliers. There were no group differences in any of these parameters (Supplementary Figure 2). Including the motion parameters as covariates in hypothesis testing did not alter the study findings.

\section{Hypothesis Testing}

Each participant's DMN functional connectivity map (ie, the DMN component from ICA), served as the dependent variable in second-level, random effects factorial models with group (ie, high- vs low-risk) as the independent variable. Age, sex, generation, familial relatedness based on a kinship coefficient (Blumenthal and Cannon-Albright, 2008), prior medication exposure, and history of depression, anxiety, or substance use disorder were included as covariates. Regions with positive functional connectivity with the DMN component were indexed within DMN connectivity; conversely, we examined connection strength between the DMN component and the CEN based on predefined masks (Seeley et al, 2007). To control for multiple statistical comparisons, for any cluster to be considered statistically significant, the cluster had to contain at least 25 neighboring voxels, with each voxel in the cluster meeting an $\alpha$ of 0.01 . The combined application of a voxel level statistical threshold and cluster filter minimizes the false-positive identification of regions at any given threshold (Forman et al, 1995) because clustering can distinguish between true connectivity between regions and noise that has less tendency to cluster (Posner et al, 2014).

\section{Exploratory Analyses}

Probabilistic tractography. To examine structural connectivity of the DMN and DMN-CEN circuitry, we performed probabilistic tractography on diffusion MRI data, as described elsewhere (Cha et al, 2015). Diffusion MRI data were processed with the FMRIB's Diffusion Toolbox (Smith et al, 2004) in FSL 5.0. The preprocessing pipeline includes skull stripping, eddy current correction, B-matrix rotation, affine registration of the $\mathrm{T} 1$-weighted, diffusion-weighted images, and FreeSurfer segmentation and parcellation images. Multifiber probabilistic diffusion modeling was performed next using a Bayesian Estimation of Diffusion Parameters Obtained using Sampling Techniques with Crossing Fibers modeling (bedpostx) program (Behrens et al, 2007). To maximize accuracy of Markov Chain Monte Carlo sampling, a burn-in sample size was set conservatively to 1000 . White matter tracts were then estimated using Probabilistic Tracking with Crossing Fibers (probtrackx2) in FSL 5.0 (Behrens et al, 2003). We used each individual's precuneus segmentation mask (conducted with FreeSurfer) as a seed (each hemisphere separately) and two dorsal prefrontal ROIs as target regions. We paid special attention to avoid false positive tracts in our tractography analyses. Specifically, we first created an exclusion mask from each individual's segmentation masks consisting of the cerebral spinal fluid and all the ventricles. In addition, to effectively account for gyral and sulcal configurations when estimating tracts going through different cortices, we created a binary mask by inverting each individual's cortical parcellation in the FreeSurfer suite. Any tracts arriving at this exclusion mask were discarded. Five thousand streamline samples were generated for each tractography run from each ROI voxel to build a connectivity distribution. We calculated the number of streamline samples from the seed mask, successfully arriving at the target ROI mask proportional to the total number of samples. We first used individual's cortical parcellation masks as the target ROIs and then excluded ROIs whose probabilistic measures did not reach a threshold of $0.02 \%$ of the total estimated streamlines; this is a commonly used threshold in prior studies (Forstmann et al, 2011; Li et al, 2012; Chowdhury et al, 2013). Thus, the connectivity measures represent ROI-to-ROI probabilistic connectivity. Tractography analyses were performed on a Linux-based high-performance computing system at Columbia University's Advanced Research Computing Services. For group-wise comparisons, we used factorial models analogous to those used in our hypothesis testing and restricted the tractography analysis to connections in which group differences were detected during hypothesis testing. We used false discovery rate (FDR) to correct for multiple comparisons.

Path analyses. We used path analysis to test two models: (i) $\mathrm{DMN}$ connectivity mediating a relationship between familiar risk for depression and depressive symptoms and (ii) DMN-CEN connectivity mediating a relationship between familiar risk for depression and impulsivity. Following established methods for mediation (Rucker et al, 2011), the path analyses were conducted using a series of linear regression models. For DMN connectivity, we tested whether familial history of depression (independent variable) influenced DMN connectivity (dependent variable). In a second regression model, we then tested whether DMN connectivity (independent variable) influenced depressive symptoms (dependent variable) while controlling for family 
history. For DMN-CEN connectivity, the same analytic approach was used except DMN-CEN connectivity was the dependent variable in the first regression model and impulsivity was the dependent variable in the second regression model. Impulsivity was assessed with the Continuous Performance Task II commission errors (CPT-II, Commissions; Conners and Staff, 2000). A complete description of the CPT-II is provided in the Supplementary Materials. The path analyses were conducted using SPSS (SPSS Inc., Chicago, IL). DMN and DMN-CEN connectivity were extracted from SPM (http://www.fil.ion.ucl.ac.uk/spm/). Statistical significance was determined using the Sobel test. Age and sex were included as covariates.

Sensitivity analyses. Potential confounds of the study were considered. First, anxiety symptoms were greater in the adults within the high- $v$ s low-risk group (Table 1). Second, although all study participants were examined using the same MRI platform, 11 of the study participants (6 from the high-risk group and 5 from the low-risk group) were scanned at a different site because of renovations at the study's primary site (although both sites used a GE Signa 3.0 T, whole-body scanner, 8-channel head coil). Third, the number of ICA-components generated for each individual could influence our hypothesis testing. To address the first potential confounds, we added the following covariates to our hypothesis testing: anxiety and depressive symptom severity. Anxiety symptoms were assessed with the Hamilton Anxiety Rating Scale and the Revised Children's Manifest Anxiety Scale for adults and children, respectively. Depression symptoms were assessed with the Hamilton Rating Scale for Depression (Hamilton, 1960) and the Children's Depression Rating Scale-Revised (Poznanski and Mokros, 1996) for adults and children, respectively. Because the assessment measures for depressive and anxiety symptoms differed in adults $v s$ children, symptom severity scores were transformed into $\mathrm{z}$-scores. Second, to exclude the possibility that the study findings were confounded by differences in MRI scanners, we excluded the 11 participants who were scanned at the alternate site. These sensitivity analyses did not meaningfully alter the study findings (Supplementary Materials). Third, the total number of ICA-components generated for each individual did not differ between the two groups $(p=0.6)$. Moreover, subgroup analysis excluding participants with ICA-components outside of the 95\% CI of the group mean did not alter our hypothesis testing (Supplementary Materials).

\section{RESULTS}

\section{Clinical Measures}

Depression and anxiety disorders were more common in the high- $v s$ low-risk group, as were current anxiety symptoms (Table 1). Relative to the low-risk group, the high-risk group had more G2 than G3 participants. Including these variables as covariates did not meaningfully alter the study findings.

\section{Hypothesis Testing: Functional Connectivity Measures}

ICA with a partner matching hierarchical algorithm isolated the DMN in both groups. Connectivity maps demonstrated the commonly observed connectivity pattern of the DMN with significant connectivity detected within the posterior cingulate cortex, precuneus, medial prefrontal cortex, lateral parietal cortex bilaterally, and superior frontal cortex bilaterally. In both groups, we also detected anticorrelations between the DMN and regions within the CEN including the dorsolateral prefrontal cortex (DLPFC) and parietal cortex (Figure 1b).

Compared with low-risk participants, high-risk participants showed increased DMN connectivity within DMN regions including the precuneus/posterior cingulate and left lateral parietal cortex (Figure 1a and Table 2). There were no regions of increased DMN connectivity in the low-risk group. Compared with the low-risk participants, high-risk participants also showed reduced DMN-CEN-negative connectivity (ie, reduced anticorrelations) with CEN regions including the anterior portion of the DLPFC, bilaterally (Figure $1 \mathrm{~b}$ and Table 2). There were no regions of reduced DMN-CEN-negative connectivity in the low-risk group. Controlling for current or prior depressive, anxiety, substance use disorders, generation, or motion parameters did not meaningfully alter the DMN or DMN-CEN connectivity findings, nor did excluding participants with a current or lifetime history of depression. To further explore effects of age, we ran additional analyses. We compared the high- vs low-risk participants within specific age ranges: (i) under 18 years old; (ii) $18-25$ years old; (iii) $26-45$ years old; and (iv) over 45 years old. Regardless of the age range, the highrisk participants showed increased DMN connectivity and reduced DMN-CEN-negative connectivity (Supplementary Materials).

\section{Exploratory Analyses: Tractography}

Precuneus seeded white matter tracts projected to the frontal gyrus primarily through cingulum bundles (Supplementary Figure 1). Inter-hemispheric connections through major and minor forceps were apparent. Compared with low-risk participants, high-risk participants showed significantly decreased probabilistic tract measures between the precuneus and the right DLPFC $\left(P_{\mathrm{FDR}}=0.048\right.$; general linear model controlling for gender, age, and motion parameters of diffusion weighted images; Supplementary Figure 1). Other tracts show non-significant group effects at FDR adjusted $P$-value of 0.05 . In relation to the functional connectivity results, robust regression revealed a significant correlation between (i) the tract measures between the precuneus and DLPFC and (ii) the DMN functional connectivity with the DLPFC $\left(t=-2.61, P_{\mathrm{FDR}}=0.04\right.$; robust regression controlling for gender, age, and motion parameters).

\section{Exploratory Analyses: Path Analysis}

Using path analysis, we examined whether a relationship between familial risk and depressive symptoms might be mediated by increased DMN connectivity. We did not find evidence for a mediation effect.

In a second path analysis, we examined whether a relationship between familial risk and impulsivity might be mediated by decreased negative DMN-CEN connectivity. We restricted this analysis to regions in which the highrisk group demonstrated decreased negative DMN-CEN 
a

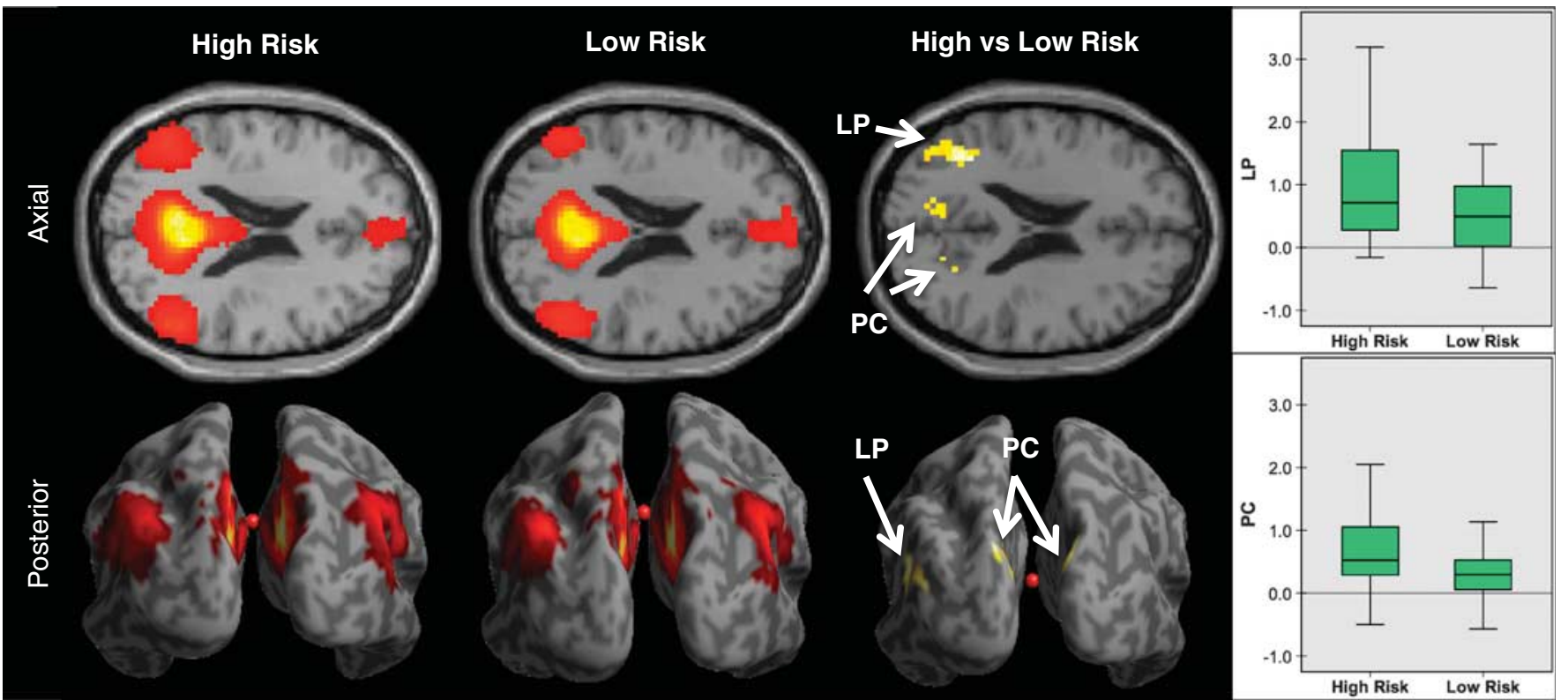

b
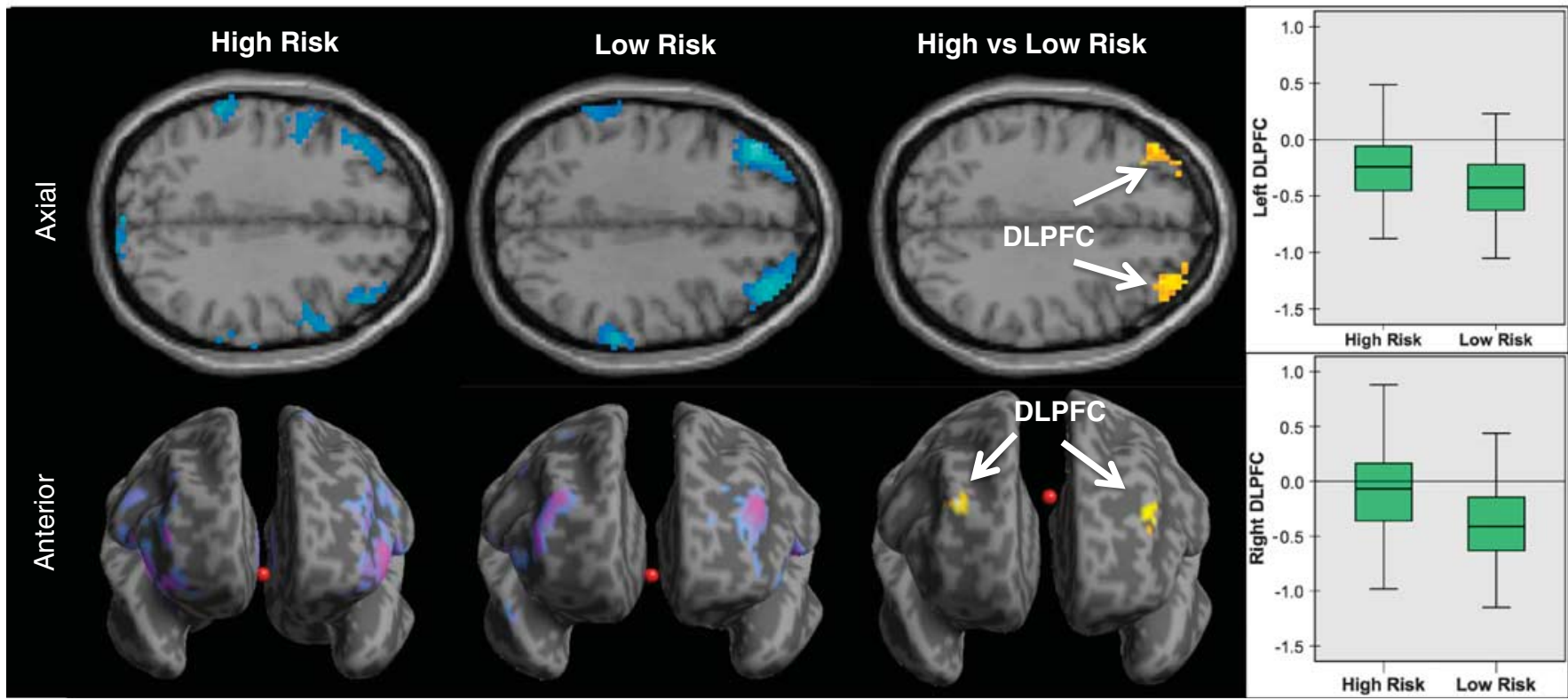

Figure I Familial risk for depression and default mode network connectivity. (a) Whole-brain resting-state functional-connectivity maps of the default mode network (DMN). The connectivity maps demonstrate the commonly observed connectivity pattern of the DMN in both the high- and low-risk groups. Orange-red indicates positively correlated fMRI signal, or positive connectivity, within regions of the DMN. Comparison of the groups (high- vs low-risk) demonstrated that the participants in the high-risk group had stronger DMN connections at the lateral parietal lobe (LP) and the precuneus (PC). Boxplot shows the mean connectivity strengths (z-scores) for both groups in the LP and PC. (b) Whole-brain resting-state functional-connectivity maps of the DMN with the task-positive regions including the central executive network (CEN). Blue-purple indicates inversely correlated fMRI signal, or anticorrelations, with the DMN. Comparison of the groups (high- vs low-risk) demonstrated that the participants in the high-risk group had weaker DMN-CEN anticorrelations (or less inversely correlated $\mathrm{PMRI}$ signal) at the anterior portion of the dorsolateral prefrontal cortex (DLPFC), bilaterally. Boxplot shows the mean connectivity strengths (z-scores) for both groups in the right and left DLPFC.

connectivity during hypothesis testing. In the first linear regression model, we found family history to be a significant predictor of decreased negative DMN-CEN connectivity at the right DLPFC $(\beta=0.35, t=4.07, P<0.001)$ and the left DLPFC ( $\beta=0.24, t=3.01, P=0.003)$. We then used a second linear regression model and found that while controlling for family history, decreased negative DMN-CEN connectivity at the right DLPFC was a significant predictor of impulsivity as measured by CPT-II, commissions $(\beta=3.73, t=2.44$, $P=0.017)$. Decreased negative DMN-CEN connectivity at the left DLPFC was also a significant predictor of impulsivity $(\beta=3.82, t=2.31, P=0.023)$. Last, we used the Sobel test to confirm the significance of the indirect effect of familial risk on impulsivity as mediated by DMN-right DLPFC 
Table 2 Functional Connectivity in Individuals at High and Low Risk for Depression

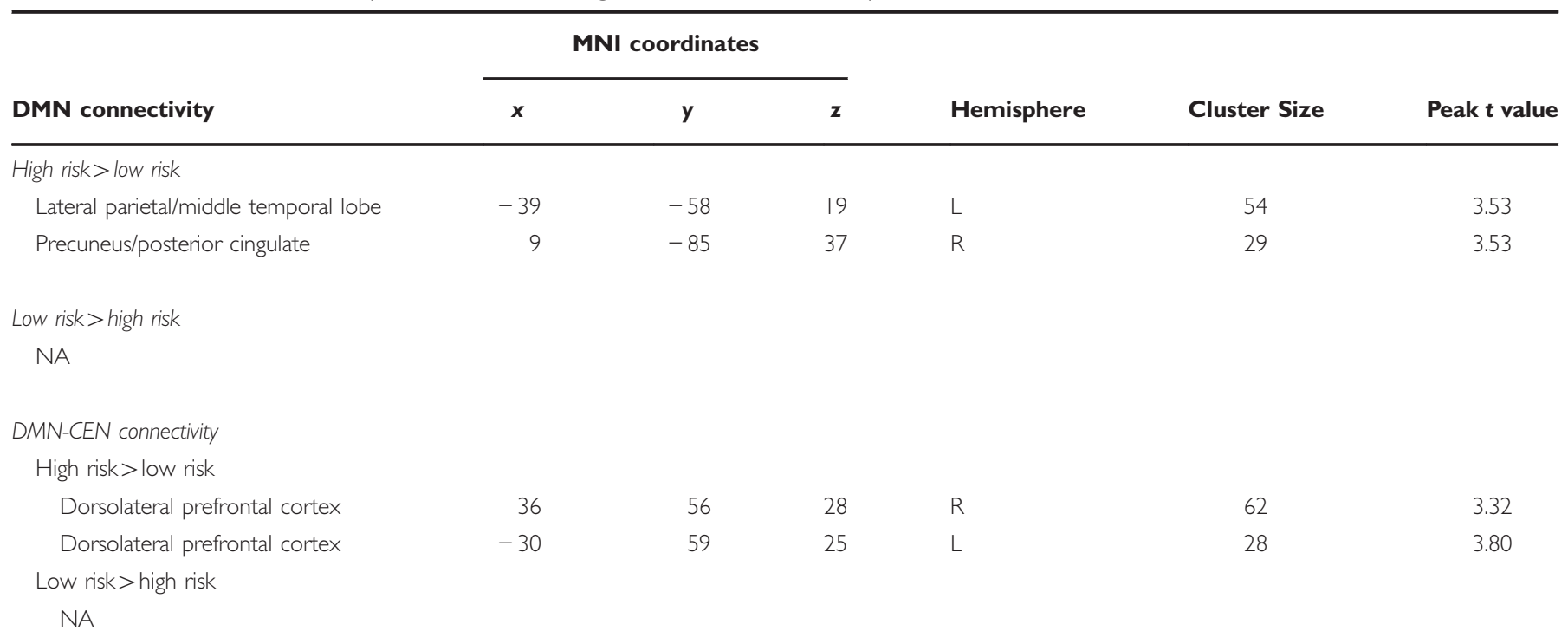

Abbreviations: L, left; R, right; NA, not applicable; MNI, Montreal Neurological Institute. Cluster size in $3 \mathrm{~mm}^{3}$ voxels; covariates: age, sex, generation, familial relatedness, prior medication exposure, and history of depression, anxiety, and substance use disorder. Age, sex, generation, familial relatedness based on a kinship coefficient (Blumenthal and Cannon-Albright, 2008).

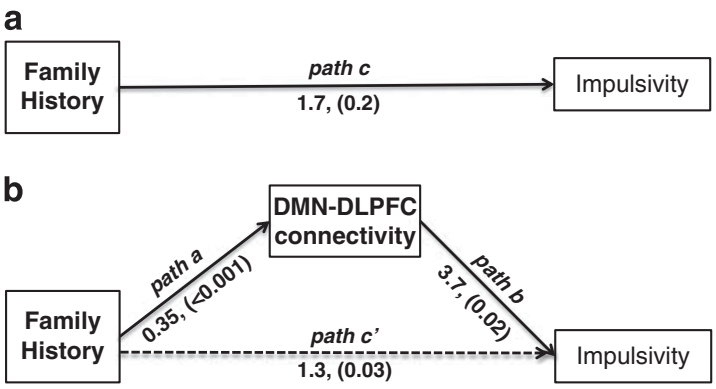

Figure 2 Path modeling of default mode network connectivity. (a) There was a non-significant effect of family history on impulsivity (path $c, P=0.2$ ). (b) However, an indirect effect was detected (indicated by dotted line) indicating that connectivity between the default mode network (DMN) and the right dorsolateral prefrontal cortex (DLPFC) mediated an effect of family history on impulsivity. In the first linear regression model, we found family history to be a significant predictor of decreased negative DMN-CEN connectivity at the right DLPFC (path $a, \beta=0.35, t=4.07, P<0.00 \mathrm{I}$ ). We then used a second linear regression model and found that while controlling for family history, decreased negative DMN-CEN connectivity at the right DLPFC was a significant predictor of impulsivity as measured by CPT-II, commissions (path $b, \beta=3.73, t=2.44, P=0.017$ ). Last, we used the Sobel test to confirm the significance of the indirect effect of familial risk on impulsivity as mediated by DMN-CEN connectivity (path c', coefficient $=1.3, P=0.03$ ). DMN-CEN connectivity at the left DLPFC was also a mediator of family history and impulsivity (coefficient $=0.9, P=0.05$ ).

connectivity (coefficient $=1.3 P=0.03$, Figure 2 ) and DMNleft DLPFC connectivity (coefficient $=0.9, P=0.05$ ). Reverse mediation effects were not significant (ie, impulsivity did not mediate effects of familial risk on DMN-CEN hyperconnectivity). We did not detect a direct relationship between familial risk and impulsivity; however, a direction relationship between outcome (ie, impulsivity) and predictor (familial risk) is not required for path-based mediation analyses and is often not detected (Zhao et al, 2010; Rucker et al, 2011).

\section{DISCUSSION}

We examined DMN connectivity in individuals at high and low familial risk for MDD. We found that relative to individuals at low familial risk, those at high risk had increased DMN connectivity as well as decreased negative DMN-CEN connectivity (ie, decreased DMN-CEN anticorrelations). These findings were evident even after excluding participants with a current or lifetime history of MDD, suggesting that the connectivity findings are precursors rather than the consequence of MDD. Exploratory analyses with diffusion tractography corroborated the finding of decreased negative DMN-CEN functional connectivity in high-risk individuals. Path analyses suggested that the decreased negative DMN-CEN connectivity mediates an effect of familial risk on impulsivity.

Prior studies suggest that individuals with depressive disorders have altered functionality and connectivity of the DMN (Greicius et al, 2007; Sheline et al, 2010; WhitfieldGabrieli and Ford, 2012). Functional MRI studies, for example, indicate that in patients with depressive disorders, the DMN is hyperactive during periods self-referential thought (Sheline et al, 2009), and this increased activity does not abate during attention-demanding tasks (Anticevic et al, 2012; Wagner et al, 2013). Individuals with remitted depression also demonstrate this failure to suppress DMN activity during attention-demanding tasks (Marchetti et al, 2012; Bartova et al, 2015). Similarly, when individuals with or without depression engage in self-referential thinking, DMN connectivity increases; conversely, as individuals shift from self-referential to externally focused thoughts, DMN 
connectivity reduces in healthy controls but remains increased in depressed individuals (Belleau et al, 2014). Studies examining the resting state with fMRI demonstrate hyperconnectivity within the DMN in patients with MDD (Greicius et al, 2007), dysthymic disorder (Posner et al, 2013), and remitted depression (Nixon et al, 2014). Antidepressants reduce DMN hyperconnectivity in depressed patients to levels comparable with healthy control participants (Posner et al, 2013), and reductions in prefrontal connectivity correlate with symptom improvement (Wang et al, 2015). In adolescents with depression, altered connectivity has been reported between the amygdala and prefrontal cortex (Cullen et al, 2014), as well as between the amygdala and the anterior cingulate cortex (Connolly et al, 2013; Ho et al, 2014).

Our findings build upon the prior literature on the DMN and depression by demonstrating that individuals at high familial risk for MDD have increased DMN connectivity. This finding remained evident after excluding participants with a current or lifetime history of MDD. By examining individuals at risk, but not yet affected by depression, this study takes a critical step in establishing that increased DMN connectivity is a precursor of MDD and may therefore indicate a biomarker predictive of the development of depression. In the context of depression, DMN hyperactivity and hyperconnectivity may reflect an excessive focus on one's own emotional state, potentially in the form of depressive ruminations (Berman et al, 2011; Posner et al, 2013). Disproportionate focus on internal, emotional states with related DMN hyperconnectivity may underlie a predilection for depression in high-risk individuals. Although our study did not include a detailed assessment of ruminations (Nolan et al, 1998), future studies could examine whether preventive interventions aimed at high-risk individuals reduce DMN hyperconnectivity, diminish ruminations, and thereby avert the development of depression.

Inversely correlated fMRI signal (ie, anticorrelations) between the DMN and CEN has been described in numerous studies of healthy individuals (Fox et al, 2005; Uddin et al, 2009; Whitfield-Gabrieli and Ford, 2012). In individuals at high risk for depression, we found decreased DMN-CENnegative connectivity, or weaker anticorrelations, and similar findings have previously been reported in mood and anxiety disorders (Whitfield-Gabrieli and Ford, 2012). Specifically, we found decreased negative connectivity between the DMN and the anterior portion of the DLPFC, bilaterally, in highrisk individuals. The DLPFC is a central node within the $\mathrm{CEN}$ and is thought to subserve executive functions including inhibitory control (Fuster, 2001). This putative role of the DLPFC is consistent with the results of our path analyses. We found that weaker anticorrelations between the DMN and the DLPFC mediated impulsivity as indexed by the CPT-II. This is a striking finding given recent reports of weaker DMN anticorrelations with task-positive regions, such as the CEN, across a range of psychiatric disorders including ADHD (Castellanos et al, 2008), obsessivecompulsive disorder (Stern et al, 2012), bipolar disorder (Chai et al, 2011), and schizophrenia (Whitfield-Gabrieli et al, 2009). Similar findings are also reported in individuals with remitted depression (Jacobs et al, 2014). Our path analysis suggests a potential mechanism for these trans-diagnostic findings - that is, weaker DMN-CEN anti- correlations may produce heightened impulsivity, a welldescribed risk factor for multiple psychiatric disorders. Although it examined connectivity across different brain regions, a recent rs-fcMRI study similarly found that adolescent girls at familial risk for depression have reduced connectivity within prefrontal regions related to impulse control (Clasen et al, 2014). In addition, our DTI tractography results reinforced our rs-fcMRI analyses: high-risk individuals showed decreased white matter connectivity between the precuneus (a central node within the DMN) and the DLPFC, paralleling our rs-fcMRI finding of weaker DMN-CEN anticorrelations in high-risk participants.

An important alternative interpretation of the study findings merits consideration. Rather than indicating risk, the study findings could reflect resilience, or a neural adaptation against the development of depression. Individuals at high familial risk could, eg, develop altered connectivity in response to the chronic stress of developing depression. The cross-sectional nature of the MRI data collected makes it impossible to fully exclude this interpretation; however, two important factors make it unlikely: first, if increased DMN connectivity represented a marker of resilience, one would hypothesize that the high-risk group would have greater depressive symptom severity. This was not the case, and moreover, covarying for depressive symptoms did not alter the study findings. Second, prior research indicates that antidepressants reduce DMN connectivity (Posner et al, 2013). If increased DMN connectivity reflected resilience, one would hypothesize that antidepressants should increase, not reduce, DMN connectivity. These rationales notwithstanding, a longitudinal study is the best approach to fully test the hypothesis that DMN and DMN-CEN connectivity indicate biomarkers of risk for depression.

Study limitations should be noted. First, familial risk for depression is inarguably conferred through a combination of genetic loading, environmental factors, and gene-byenvironment interactions. Our study was not designed to disentangle the unique contributions of environmental $v s$ genetic effects. Second, as noted previously, a prospective analysis of the DMN and DMN-CEN findings is needed to comprehensively test the utility of the study findings as biomarkers predictive of MDD. Third, this study analyzed functional connectivity and not task-related activations. The relationship between familial risk for depression and functional connectivity of the DMN and DMN-CEN may differ from the relationship between familial risk and taskrelated activations of these networks. Indeed, in a prior report, data suggest that task-related activations of the DMN may be a marker of recovery from, rather than risk for, depression (Peterson et al, 2014). Taken together, whereas increased connectivity seems to predate the onset of depression, alterations in DMN task-related activations may occur after illness onset. Fourth, the study sample was Caucasian and thus the generalizability of the findings across racial and ethnic groups should not be assumed. Fifth, the optimal ICA methodology for making group-level inferences from resting fMRI data is still an open question (Erhardt et al, 2011). Although our approach to ICA has been previously validated (Wang and Peterson, 2008), it is nonetheless possible that our methodology influenced our findings. 
In conclusion, this is the largest study to examine restingstate functional connectivity in individuals at high and low familial risk for depression. The findings suggest that increased DMN connectivity as well as decreased DMN-CEN anticorrelations are antecedents rather than the consequence of MDD. Path analyses point to important potential mechanisms by which DMN-CEN connectivity may confer risk for MDD and other disorders. Resting-state functional connectivity may offer an important method for identifying individuals likely to develop MDD, a critical step to advancing prevention and early intervention for this major public health issue.

\section{FUNDING AND DISCLOSURE}

This study was supported in part by NIMH grants R01MH036197 (MMW) and K23-MH091249 (JP). Dr Posner is a principal investigator on an investigator-initiated grant from Shire Pharmaceuticals. The remaining authors have no competing interests to report.

\section{REFERENCES}

Anticevic A, Cole MW, Murray JD, Corlett PR, Wang X-J, Krystal JH (2012). The role of default network deactivation in cognition and disease. Trends Cogn Sci 16: 584-592.

Bartova L, Meyer BM, Diers K, Rabl U, Scharinger C, Popovic A et al (2015). Reduced default mode network suppression during a working memory task in remitted major depression. J Psychiatr Res 64: 9-18.

Behrens TE, Berg HJ, Jbabdi S, Rushworth MF, Woolrich MW (2007). Probabilistic diffusion tractography with multiple fibre orientations: What can we gain? Neuroimage 34: 144-155.

Behrens TE, Woolrich MW, Jenkinson M, Johansen-Berg $\mathrm{H}$, Nunes RG, Clare S et al (2003). Characterization and propagation of uncertainty in diffusion-weighted MR imaging. Magn Reson Med 50: 1077-1088.

Belleau EL, Taubitz LE, Larson CL (2014). Imbalance of default mode and regulatory networks during externally focused processing in depression. Social Cogn Affect Neurosci nsu117.

Berman MG, Peltier S, Nee DE, Kross E, Deldin PJ, Jonides J (2011). Depression, rumination and the default network. Social Cogn Affect Neurosci 6: 548-555.

Biswal BB, Mennes M, Zuo X-N, Gohel S, Kelly C, Smith SM et al (2010). Toward discovery science of human brain function. Proc Natl Acad Sci USA 107: 4734-4739.

Blumenthal DT, Cannon-Albright LA (2008). Familiality in brain tumors. Neurology 71: 1015-1020.

Castellanos FX, Margulies DS, Kelly C, Uddin LQ, Ghaffari M, Kirsch A et al (2008). Cingulate-precuneus interactions: a new locus of dysfunction in adult attention-deficit/hyperactivity disorder. Biol Psychiatry 63: 332-337.

Cha J, Fekete T, Siciliano F, Biezonski D, Greenhill L, Pliszka S et al (2015). Neural Correlates of Aggression in Medication Naive Children with ADHD: Multivariate Analysis of Morphometry and Tractography. Neuropsychopharmacology 40: 1717-1725.

Chai XJ, Whitfield-Gabrieli S, Shinn AK, Gabrieli JD, Castañón AN, McCarthy JM et al (2011). Abnormal medial prefrontal cortex resting-state connectivity in bipolar disorder and schizophrenia. Neuropsychopharmacology 36: 2009-2017.

Chowdhury R, Lambert C, Dolan RJ, Duzel E (2013). Parcellation of the human substantia nigra based on anatomical connectivity to the striatum. Neuroimage 81: 191-198.

Clasen PC, Beevers CG, Mumford JA, Schnyer DM (2014). Cognitive control network connectivity in adolescent women with and without a parental history of depression. Dev Cogn Neurosci 7: 13-22.

Conners CK, Staff M (2000). Conners' Continuous Performance Test II (CPT II V. 5). ' Multi-Health Systems Inc.: North Tonawanda, NY.

Connolly CG, Wu J, Ho TC, Hoeft F, Wolkowitz O, Eisendrath S et al (2013). Resting-state functional connectivity of subgenual anterior cingulate cortex in depressed adolescents. Biol Psychiatry 74: 898-907.

Cullen KR, Westlund MK, Klimes-Dougan B, Mueller BA, Houri A, Eberly LE et al (2014). Abnormal amygdala resting-state functional connectivity in adolescent depression. JAMA Psychiatry 71: 1138-1147.

Dubin M, Weissman M, Xu D, Bansal R, Hao X, Liu J et al (2012). Identification of a circuit-based endophenotype for familial depression. Psychiatry Res 201: 175-181.

Erhardt EB, Rachakonda S, Bedrick EJ, Allen EA, Adali T, Calhoun VD (2011). Comparison of multi-subject ICA methods for analysis of fMRI data. Hum Brain Mapp 32: 2075-2095.

Forman SD, Cohen JD, Fitzgerald M, Eddy WF, Mintun MA, Noll DC (1995). Improved assessment of significant activation in functional magnetic resonance imaging (fMRI): use of a clustersize threshold. Magn Reson Med 33: 636-647.

Forstmann BU, Tittgemeyer M, Wagenmakers EJ, Derrfuss J, Imperati D, Brown S (2011). The speed-accuracy tradeoff in the elderly brain: a structural model-based approach. J Neurosci 31: 17242-17249.

Fox MD, Snyder AZ, Vincent JL, Corbetta M, Van Essen DC, Raichle ME (2005). The human brain is intrinsically organized into dynamic, anticorrelated functional networks. Proc Natl Acad Sci USA 102: 9673-9678.

Friston KJ, Ashburner CD, Frith JB, Poline JB, Heather RS, Frackowiak RS (1995). Spatial registration and normalization of images. Hum Brain Mapp 3: 165-189.

Fuster J (2001). The prefrontal cortex-An update time is of the essence. Neuron 30: 319-333.

Grady CL, Protzner AB, Kovacevic N, Strother SC, Afshin-Pour B, Wojtowicz $M$ et al (2010). A multivariate analysis of age-related differences in default mode and task-positive networks across multiple cognitive domains. Cerebral Cortex 20: 1432-1447.

Greicius MD, Flores BH, Menon V, Glover GH, Solvason HB, Kenna $\mathrm{H}$ et al (2007). Resting-state functional connectivity in major depression: abnormally increased contributions from subgenual cingulate cortex and thalamus. Biol Psychiatry 62: 429-437.

Hamilton JP, Furman DJ, Chang C, Thomason ME, Dennis E, Gotlib IH (2011). Default-mode and task-positive network activity in major depressive disorder: implications for adaptive and maladaptive rumination. Biol Psychiatry 70: 327-333.

Hamilton M (1960). A rating scale for depression. Br Med J 23: 56.

Ho TC, Yang G, Wu J, Cassey P, Brown SD, Hoang N et al (2014). Functional connectivity of negative emotional processing in adolescent depression. J Affect Disorders 155: 65-74.

Jacobs RH, Jenkins LM, Gabriel LB, Barba A, Ryan KA, Weisenbach SL et al (2014). Increased coupling of intrinsic networks in remitted depressed youth predicts rumination and cognitive control. PLoS One 9: e104366.

Keller MB (2003). Past, present, and future directions for defining optimal treatment outcome in depression: remission and beyond. JAMA 289: 3152-3160.

Kessler RC, Zhao S, Blazer DG, Swartz M (1997). Prevalence, correlates, and course of minor depression and major depression in the National Comorbidity Survey. I Affect Disorders 45: 19-30.

Li L, Rilling JK, Preuss TM, Glasser MF, Hu X (2012). 'The effects of connection reconstruction method on the interregional connectivity of brain networks via diffusion tractography. Hum Brain Mapp 33: 1894-1913. 
Marchetti I, Koster EH, Sonuga-Barke EJ, De Raedt R (2012). The default mode network and recurrent depression: a neurobiological model of cognitive risk factors. Neuropsychol Rev 22: 229-251.

Nixon N, Liddle P, Nixon E, Worwood G, Liotti M, Palaniyappan L (2014). Biological vulnerability to depression: linked structural and functional brain network findings. $\mathrm{Br} J$ Psychiatry 204: 283-289.

Nolan SA, Roberts JE, Gotlib IH (1998). Neuroticism and ruminative response style as predictors of change in depressive symptomatology. Cogn Ther Res 22: 445-455.

Paradiso S, Lamberty GJ, Garvey MJ, Robinson RG (1997). Cognitive impairment in the euthymic phase of chronic unipolar depression. J Nerv Mental Dis 185: 748-754.

Peterson B, Warner V, Bansal R, Zhu H, Hao X, Liu J et al (2009). Cortical thinning in persons at increased familial risk for major depression. Proc Natl Acad Sci USA 106: 6273.

Peterson BS, Wang Z, Horga G, Warner V, Rutherford B, Klahr KW et al (2014). Discriminating risk and resilience endophenotypes from lifetime illness effects in familial major depressive disorder. JAMA Psychiatry 71: 136-148.

Posner J, Hellerstein DJ, Gat I, Mechling A, Klahr K, Wang Z et al (2013). Antidepressants normalize the default mode network in patients with dysthymia. JAMA Psychiatry 70: 373-382.

Posner J, Marsh R, Maia TV, Peterson BS, Gruber A, Simpson HB (2014). Reduced functional connectivity within the limbic corticostriato-thalamo-cortical loop in unmedicated adults with obsessivecompulsive disorder. Hum Brain Mapp 35: 2852-2860.

Posner J, Park C, Wang Z (2014). Connecting the dots: a review of resting connectivity MRI studies in attention-deficit/hyperactivity disorder. Neuropsychol Rev 24: 3-15.

Posner J, Rauh V, Gruber A, Gat I, Wang Z, Peterson BS (2013). Dissociable attentional and affective circuits in medication-naïve children with attention-deficit/hyperactivity disorder. Psychiatry Res 213: 24-30.

Power JD, Barnes KA, Snyder AZ, Schlaggar BL, Petersen SE (2012). Spurious but systematic correlations in functional connectivity MRI networks arise from subject motion. Neuroimage 59: 2142-2154.

Poznanski EO, Mokros HB (1996). Children's Depression Rating Scale, Revised (CDRS-R). Western Psychological Services: Los Angeles.

Raichle M, MacLeod A, Snyder A, Powers W, Gusnard D, Shulman G (2001). A default mode of brain function. Proc Natl Acad Sci USA 98: 676.

Raichle M, Snyder A (2007). A default mode of brain function: a brief history of an evolving idea. Neuroimage 37: 1083-1090.

Rucker DD, Preacher KJ, Tormala ZL, Petty RE (2011). Mediation analysis in social psychology: Current practices and new recommendations. Soc Personal Psychol Compass 5: 359-371.

Rush A, Trivedi M, Wisniewski S, Nierenberg A, Stewart J, Warden D et al (2006). Acute and longer-term outcomes in depressed outpatients requiring one or several treatment steps: a STAR ${ }^{\star}$ D report. Am J Psychiatry 163: 1905-1917.
Seeley WW, Menon V, Schatzberg AF, Keller J, Glover GH, Kenna $\mathrm{H}$ et al (2007). Dissociable intrinsic connectivity networks for salience processing and executive control. J Neurosci 27: 2349-2356.

Sheline YI, Barch DM, Price JL, Rundle MM, Vaishnavi SN, Snyder AZ et al (2009). The default mode network and selfreferential processes in depression. Proc Natl Acad Sci USA 106: 1942.

Sheline YI, Price JL, Yan Z, Mintun MA (2010). Resting-state functional MRI in depression unmasks increased connectivity between networks via the dorsal nexus. Proc Natl Acad Sci USA 107: 11020.

Smith SM, Jenkinson M, Woolrich MW, Beckmann CF, Behrens TE, Johansen-Berg $\mathrm{H}$ et al (2004). Advances in functional and structural MR image analysis and implementation as FSL. Neuroimage 23: S208-S219.

Stern ER, Fitzgerald KD, Welsh RC, Abelson JL, Taylor SF (2012). Resting-state functional connectivity between fronto-parietal and default mode networks in obsessive-compulsive disorder. PLoS ONE 7: e36356.

Uddin LQ, Clare Kelly A, Biswal BB, Xavier Castellanos F, Milham MP (2009). Functional connectivity of default mode network components: correlation, anticorrelation, and causality. Hum Brain Mapp 30: 625-637.

Wagner G, Koch K, Schachtzabel C, Peikert G, Schultz CC, Reichenbach JR et al (2013). Self-referential processing influences functional activation during cognitive control: an fMRI study. Soc Cogn Affect Neurosci 8: 828-837.

Wang L, Xia M, Li K, Zeng Y, Su Y, Dai W et al (2015). The effects of antidepressant treatment on resting-state functional brain networks in patients with major depressive disorder. Hum Brain Mapp 36: 768-778.

Wang Z, Maia TV, Marsh R, Colibazzi T, Gerber A, Peterson BS (2011). The neural circuits that generate tics in Tourette's syndrome. Am J Psychiatry 168: 1326-1337.

Wang Z, Peterson BS (2008). Partner-matching for the automated identification of reproducible ICA components from fMRI datasets: Algorithm and validation. Hum Brain Mapp 29: 875-893.

Weissman MM, Wickramaratne P, Nomura Y, Warner V, Verdeli H, Pilowsky DJ et al (2005). Families at high and low risk for depression: a 3-generation study. Arch Gen Psychiatry 62: 29-36.

Whitfield-Gabrieli S, Ford JM (2012). Default mode network activity and connectivity in psychopathology. Ann Rev Clin Psychol 8: 49-76.

Whitfield-Gabrieli S, Thermenos HW, Milanovic S, Tsuang MT, Faraone SV, McCarley RW et al (2009). Hyperactivity and hyperconnectivity of the default network in schizophrenia and in first-degree relatives of persons with schizophrenia. Proc Natl Acad Sci USA 106: 1279.

Zhao X, Lynch JG, Chen Q (2010). Reconsidering Baron and Kenny: Myths and truths about mediation analysis. J Consum Res 37: 197-206.

Supplementary Information accompanies the paper on the Neuropsychopharmacology website (http://www.nature.com/npp) 